\title{
Nuevos registros de Thripidae (Thysanoptera: Terebrantia] del noroeste de Argentina
}

\author{
New Records of Thripidae [Thysanoptera: Terebrantia] from \\ Northwestern Argentina
}

D.0.I.: https://doi.org/10.30550/j.azl/2018.62.2/1

\author{
María Inés Zamar ${ }^{1,4 *}$, Claudia Funes², Daniel Kirschbaum², Silvia Tapia ${ }^{3}$, \\ Gabriela Alejo ${ }^{4}$ \\ 1 Instituto de Biología de la Altura, Universidad Nacional de Jujuy. Av. Bolivia 1661, (Y4600GNE) San \\ Salvador de Jujuy, Jujuy, Argentina. Correo electrónico: mizamar25@gmail.com \\ 2 INTA Famaillá. Ruta Provincial 301, Km 32, (4132) Famaillá, Tucumán, Argentina. \\ 3 INTA EEAT Yuto, Ruta Nacional 34, Km 1286, (4518) Yuto, Jujuy, Argentina. \\ 4 Instituto de Ecorregiones Andinas (INECOA), Universidad Nacional de Jujuy, CONICET, Av. Bolivia 1661 \\ (Y4600GNE] San Salvador de Jujuy, Jujuy, Argentina.
}

Resumen - Este trabajo proporciona cuatro nuevos registros de Thripidae para Argentina, pertenecientes a Sericothripinae (Neohydatothrips portoricensis (Morgan) y Neohydatothrips hemileucus (Hood]) y Thripinae [Chaetisothrips striatus Hood y Scirtidothrips torquatus Hood]. Los ejemplares fueron recolectados en provincias del noroeste argentino (Tucumán y Jujuy). Se describen e ilustran sus características morfológicas generales, y se presentan datos sobre la distribución y plantas hospederas de cada especie.

Palabras clave: Interacciones planta-animal, biodiversidad, plantas hospederas, Sericothripinae, Thripinae.

Abstract - This work provides four new records of Thripidae in Argentina which belong to Sericothripinae (Neohydatothrips portoricensis (Morgan) and Neohydatothrips hemileucus (Hood)] and Thripinae (Chaetisothrips striatus Hood and Scirtidothrips torquatus Hood). Specimens were collected in northwestern provinces of Argentina (Tucumán and Jujuy). Their general morphological characteristics are described and illustrated, as well as information on their distribution and the host plants for each species are presented.

Keywords: Plant-animal interactions, biodiversity, host plants, Sericothripinae, Thripinae.

> Ref. bibliográfica: Zamar, M. I.; Funes, C.; Kirschbaum, D.; Tapia S.; Alejo, G. 2018. Nuevos registros de Thripidae (Thysanoptera: Terebrantia) del noroeste de Argentina. Acta zoológica lilloana 62 (2): $1-11$.

> Recibido: 15/02/18 - Aceptado: 03/08/18

$>$ URL de la revista: http://actazoologica.lillo.org.ar

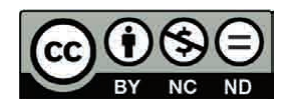




\section{INTRODUCCIÓN}

Thripidae ocupa el segundo lugar de importancia en el orden Thysanoptera, después de Phlaeothripidae. Comprende 2109 especies descriptas distribuidas en 289 géneros, con representantes en distintas regiones del mundo. Muchas especies tienen hábitos antófilos, aunque una gran proporción se reproduce sólo en hojas, algunas son depredadoras de otros artrópodos pequeños y muy pocas están asociadas con musgos. La mayoría de los trips considerados plagas de la agricultura y todos los vectores de Tospovirus son miembros de esta familia (Mound y Marullo, 1996; Commonwealth Scientific and Industrial Research Organization, 2009).

Desde la publicación del primer catálogo de Thysanoptera de Argentina por De Santis, De Sureda, Merlo (1980), quienes citaron 28 géneros y 54 especies de Thripidae, los aportes al conocimiento de esta familia han permitido incrementar su diversidad a 35 géneros y 67 especies (Goane, Pereyra, Salas, 2007; de Borbón 2008, 2009, 2013; Zamar, 2011; Zamar, et al., 2014b; Zamar, de Borbón, Aguirre, Miño, Cáceres, 2014a; Zamar, Alejo, Gutiérrez, 2015; Carrizo y Zamar, 2016).

El objetivo de este trabajo es dar a conocer cuatro nuevos registros de Thripidae para Argentina pertenecientes a las subfamilias Sericothripinae (Neohydatothrips portoricensis (Morgan) y Neohydatothrips hemileucus (Hood)) y Thripinae (Chaetisothrips striatus (Hood), y Scirtidothrips torquatus Hood), recolectadas en las provincias de Jujuy y Tucumán.

\section{MATERIALES Y MÉTODOS}

Los ejemplares estudiados provienen de muestreos realizados en las provincias de Jujuy y Tucumán. La identificación se realizó sobre la base de preparaciones microscópicas según la técnica de Mound y Marullo (1996). Los especímenes fueron examinados bajo microscopio Nikon Optiphot-2. Cada especie tratada se acompaña de una breve diagnosis, plantas hospederas, datos de colección e imágenes fotográficas de los carac- teres morfológicos externos que permitieron su clasificación.

El material se encuentra depositado en la Colección Entomológica del Instituto de Biología de la Altura de la Universidad Nacional de Jujuy (INBIAL). Los números de colección se indican en el material examinado.

\section{RESULTADOS Y DISCUSIÓN}

\section{THRIPIDAE SERICOTHRIPINAE}

Esta subfamilia se encuentra integrada por los géneros Neohydatothrips John, Hydatothrips Karny y Sericothrips Haliday, que se distinguen de otros Thripidae por presentar la cabeza rectangular, con tres pares de setas ocelares; antenas formadas por ocho segmentos, III y IV con un cono sensorial bifurcado, cada uno, VI con un cono sensorial simple elongado y externo; fémures y tibias anteriores con hileras transversas de microtriquias; el pronoto lleva generalmente una mancha de color más oscuro que la coloración del cuerpo; mesoesterno con espínula; primera vena de las alas anteriores con una hilera continua de setas, segunda vena sin setas o con una o dos cercanas al ápice; tercio lateral de los tergos abdominales II-VII con hileras de microtriquias muy próximas entre sí; peine del tergo VIII, completo; tergo IX con al menos cuatro pares de setas posteromarginales conspicuas y dos pares medio dorsales.

Las especies de Sericothripinae pueden encontrarse en flores o en hojas, pero se desconoce la biología precisa de la mayoría de ellas (CSIRO, 2009).

\section{NEOHYDATOTHRIPS JOHN}

Lima y Mound (2016) actualizaron el conocimiento de las especies de Neohydatothrips para la región Neotropical e incluyeron 41 especies, de las cuales, solo Neohydatothrips burungae (Hood), Neohydatothrips lassatus (De Santis) y Neohydatothrips sidae. (Crawford), están confirmadas para Argentina. Esta última fue recolectada en Tucumán sobre Solanum nigrum L. y Bidens pilosa L. (Carrizo y Zamar, 2016). La contribución de 
Zamar et al. (2014b) ha incluido también para el país a Neohydatothrips samayunkur (Kudo). Las citas nuevas de $N$. hemileucus y $N$. portoricencis, incrementan en seis el número de especies de este género en Argentina.

\section{NEOHYDATOTHRIPS HEMILEUCUS} (HoOD)

Hembra macróptera (Fig. 1A). Cuerpo de coloración blanquecina, excepto la cabeza, mesonoto y metanoto, segmentos abdominales II-IV y VII y zona central del tergo VIII, que son de color castaño; alas anteriores con tres bandas castañas, ápice blanquecino, clavus castaño; patas blanquecinas excepto el lado interior en las tibias anteriores y mitad distal de los fémures; segmentos antenales I y II blanquecinos, II-IV, blanquecinos con el cuarto distal castaño claro, VI-VIII, castaño claro. Setas de color castaño oscuro. Cabeza estriada, las líneas muy próximas entre sí, sin marcas entre ellas; apodema occipital nítido, separado de los ojos; tres setas postoculares, la primera, larga y gruesa, la segunda diminuta y la tercera pequeña (Fig. 1C). Segmentos antenales III y IV alargados con una constricción basal (Fig. 1E). Pronoto estriado, sin marcas entre las líneas; se destacan zonas circulares lisas en los extremos de la serie transversal de setas, otra formando un banda debajo de las setas y dos ubicadas arriba de las bases de las setas posteromarginales (Fig. 1D). Mesonoto y metanoto, estriados (Fig. 1F). Primera vena de las alas anteriores con 19 setas, las 4-5 primeras, ubicadas en la banda castaño central, segunda vena, sin setas. Margen posterior de los tergos I-VI con peine lateral, tergo VII con peine completo y dientes largos (Fig. 1G).

Macho macróptero (Fig. 1B). Similar a la hembra, puede presentar los tergos VII y VIII de color castaño o solo el VIII.

Plantas hospederas. - No existen referencias sobre la biología de esta especie. Fue recolectada en Brasil, de especies de plantas de distintas familias como Balfourodendron riedelianum (Engl.) Engl. (Rutaceae), y Dra- cena marginata Baker (Asparagaceae) (Lima y Mound, 2016). En este estudio N. hemileucus fue obtenida a partir de una trampa de caída ubicada en la vegetación espontánea presente en un campo de cultivo de crisantemos.

Material examinado.- Argentina, Jujuy, El Carmen, Las Pampitas (24 23'45,59' S; 6503'33,52" O), 1 hembra, 14/X/2016, 00018 INBIAL; 1 macho, 14/X/16, 00019 INBIAL; 1 macho 01-XII-2016. 00020 INBIAL, en trampa de caída amarilla ubicada entre la vegetación espontánea en un cultivo de Chrysanthemum morifolium (Asteraceae). Col. Gabriela Alejo.

\section{NEOHYDATOTHRIPS PORTORICENSIS (MORGAN)}

Hembra macróptera (Fig. 2A). Cuerpo bicoloreado, cabeza, zona central del pronoto, mesonoto, metanoto, zonas laterales de los tergos abdominales II-V, tergos VII a $\mathrm{X}$, líneas próximas a los márgenes anteriores de los tergos II a VI, de color castaño, zona media de los tergos segmentos abdominales II a V de coloración amarillenta, tergo VI amarillento con el margen anterior castaño; alas anteriores con bandas, la media y distal, de color castaño, tibias y tarsos amarillentos; segmentos antenales I-II amarillo claro; IIIIV amarillos con el ápice castaño, V amarillo en la base; VI a VIII, castaño. Cabeza rectangular, estriada con marcas internas entre las líneas; apodema occipital próximo a margen posterior de los ojos; dos setas postoculares (Fig. 2B-C). Segmentos antenales III y IV alargados con una constricción basal (Fig. 2D). Pronoto estriado, con marcas entre las líneas; excepto en algunas zonas pequeñas circulares u oblongas laterales (Fig. 2E). Mesonoto y metanoto, estriados, con marcas entre las líneas (Fig. 2F). Primera vena de las alas anteriores con 27-28 setas, segunda vena, con dos setas distales. Setas medias de los tergos abdominales aumentan de tamaño en sentido posterior. Superficie lateral de los tergos cubiertas con líneas de microtriquias muy densas (Fig. 2G). Margen posterior de 

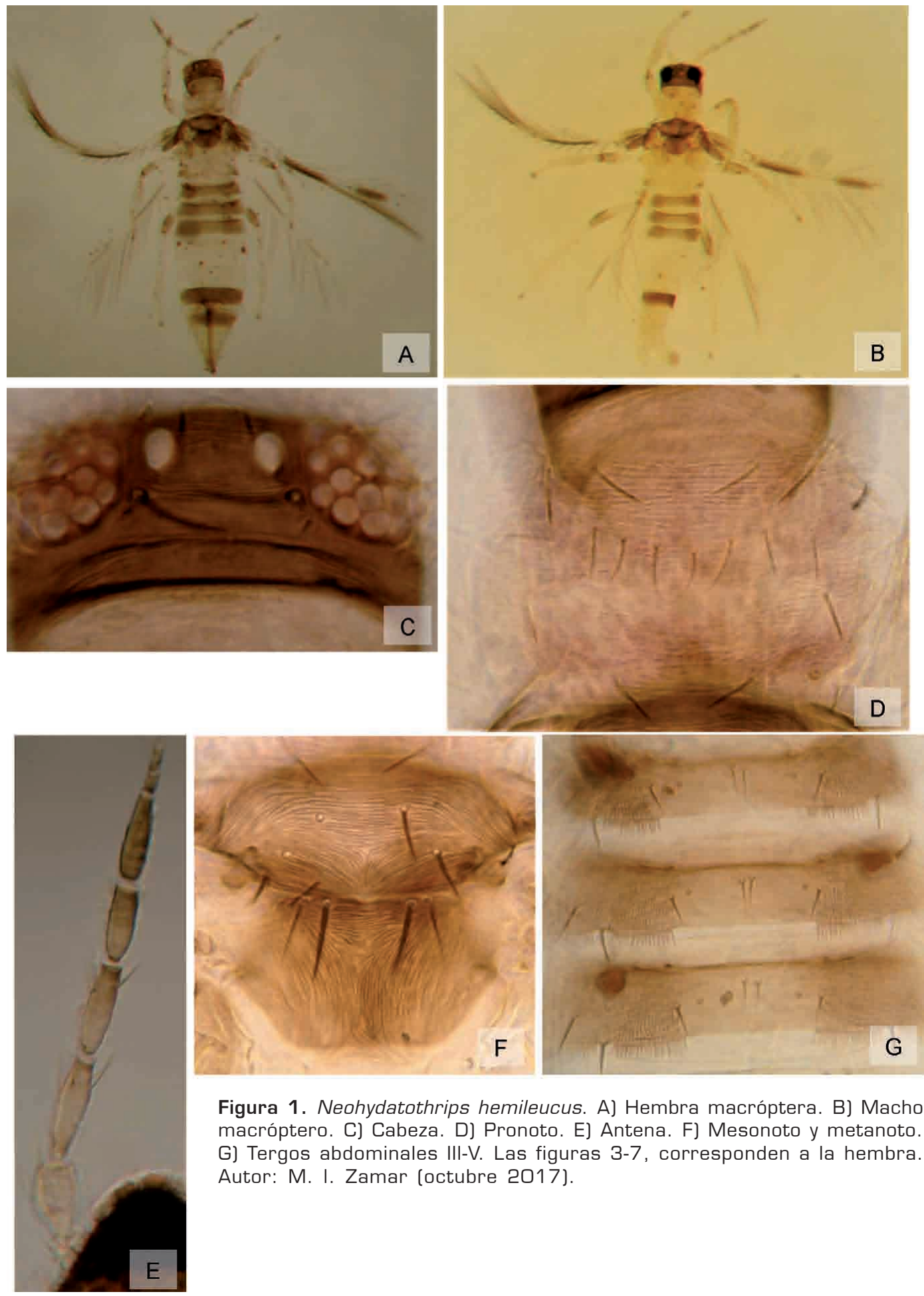

Figura 1. Neohydatothrips hemileucus. A] Hembra macróptera. B] Macho macróptero. C) Cabeza. D) Pronoto. E) Antena. FJ Mesonoto y metanoto. G) Tergos abdominales III-V. Las figuras 3-7, corresponden a la hembra. Autor: M. I. Zamar (octubre 2017). 

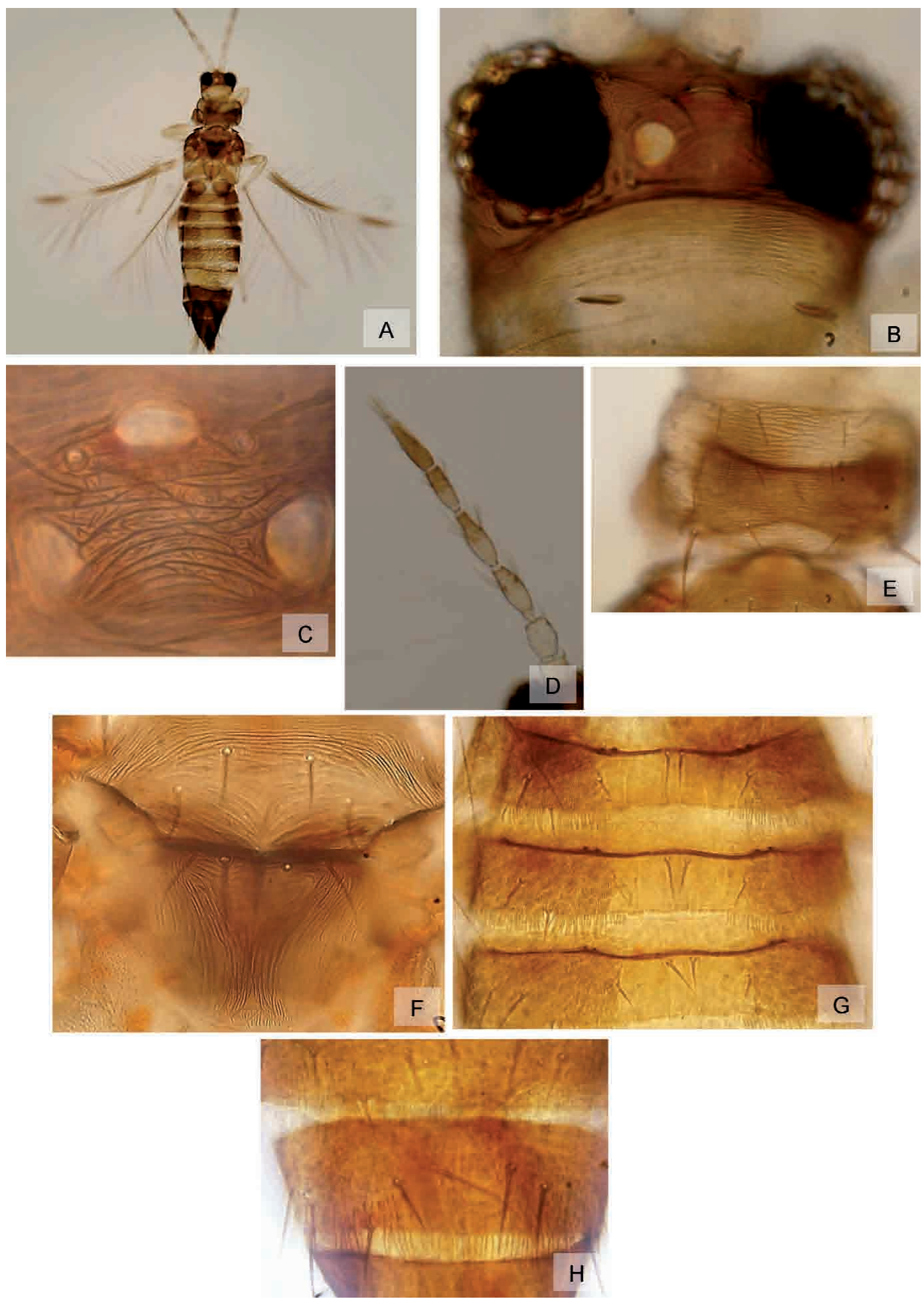

Figura 2. Neohydatothrips portoricensis. A) Hembra macróptera. B] Cabeza. C) Detalle de las estriaciones del triángulo ocelar. D) Antena. E) Pronoto. F) Mesonoto y metanoto. G) Tergos abdominales III-V. H) Peine del tergo abdominal VIII. Autor: M. I. Zamar (octubre 2017). 
los tergos II-VI con peine completo pero los dientes de la zona media son más pequeños que los de las zonas laterales (Fig. 2 G), tergos VII y VIII con peine completo y dientes largos (Fig. 2H).

Macho: no observado.

Plantas hospederas. - Al presente no existen referencias sobre la biología de esta especie. Fue obtenida a partir de hojas de Ipomoea sp. en Costa Rica y Brasil (Lima y Mound, 2016). En Argentina se recolectó mediante una trampa de caída ubicada en un cultivo de frutilla.

Material examinado.- Argentina, Tucumán, Famaillá $\left(27^{\circ} 03^{\prime} \mathrm{S}, 6^{\circ} 25^{\prime} \mathrm{O} ; 363 \mathrm{~m}\right.$ s.n.m.), 1 hembra, 27/VII/2015; 00021 INBIAL; en trampa de caída azul ubicada entre la vegetación espontánea en un cultivo de Fragaria $x$ ananassa Duch. (Rosaseae). Col. Claudia Funes.

\section{THRIPINAE}

Es la subfamilia más diversa de Thripidae, incluye 239 géneros y 1712 especies (CSIRO, 2009) pero es probable que no sea monofilética (Buckman, Mound, Whiting, 2013). La mayoría de las especies se alimentan de hojas o flores de plantas superiores, muchas son plagas agrícolas, algunas son depredadoras y muy pocas están asociadas con musgos o helechos (CSIRO, 2009).

\section{CHAETISOTHRIPS PRIESNER}

Este género contiene siete especies distribuidas en América Central y el Caribe pero solo Ch. striatus fue recolectada en Brasil (Mound y Marullo, 1996; Cavalleri, Piccoli Romanowski, Rodrigues Redaelli, 2006). Se reconoce por presentar las antenas formadas por ocho segmentos, tres pares de setas ocelares, las del primer par generalmente ubicadas una sobre la otra (Fig. 3C); las esculturaciones del pronoto consisten en numerosas líneas transversales (Fig. 3D), mesonoto y metanoto, estriados (Fig. 3E); ambas venas de las alas anteriores con un hilera completa de setas (Fig. 3G); los tergos abdominales posteriores llevan una hilera oblicua de microtriquias semejante a una ctenidia (Figs. $3 \mathrm{H}-\mathrm{I}-\mathrm{J})$; tergo VIII con el peine completo (Fig. 3J); esternos sin setas discales pero las setas marginales del esterno VII están ubicadas sobre el margen; machos con un placa porosa transversal sobre los esternos III -VII (Fig. 3K) (Mound y Marullo, 1996).

\section{CHAETISOTHRIPS STRIATUS (HOOD)}

Hembra macróptera (Fig. 3A). Cuerpo de color castaño; ocelos de base anaranjada rojiza; antenas castaño oscuro, excepto el pedicelo, cuarto basal del segmento III y bases de los segmentos IV y V, de color amarillento; tergos del tórax castaño claro; patas castaño amarillento; alas anteriores castaño con una zona sub-basal blanquecina. Cabeza estriada, con tres pares de setas ocelares, las setas I ubicadas una debajo de la otra (Fig. 3C); conos sensoriales de los segmentos III y IV, bifurcados. Pronoto estriado, excepto en algunas zonas circulares laterales y medio posteriores; dos pares de setas posteroangulares, las internas dos veces más largas que las externas y estas últimas más fuertes y largas que las discales (Fig. 3D); furcas meso y metatorácicas, bien desarrolladas (Fig. 3F). Mesonoto estriado excepto en el cuarto anterior donde presenta reticulaciones, setas medias y posteriores del mismo largo (Fig. 3E). Metanoto estriado longitudinalmente en la zona media anterior y con estrías verticales en la zona media posterior y hacia los lados; setas marginales y medias del mismo largo, las últimas, ubicadas debajo del margen anterior; sensilias campaniformes, presentes (Fig. 3E). Alas anteriores estrechas y agudas en el ápice; primera y segunda vena con series completas de setas (Fig. 3G). Tergos abdominales II a VIII, con un par de setas medias; IV a VIII con una hilera oblicua de microtriquias, las del VIII ubicadas debajo del espiráculo; estriaciones laterales del tergo II (Fig. 3H) más próximas entre sí que en el tergo IV (Fig. 3I); peine del tergo VIII, completo (Fig. 3J); margen posterior lateral de los tergos II a VII con una serie de dientes diminutos; esternos re- 

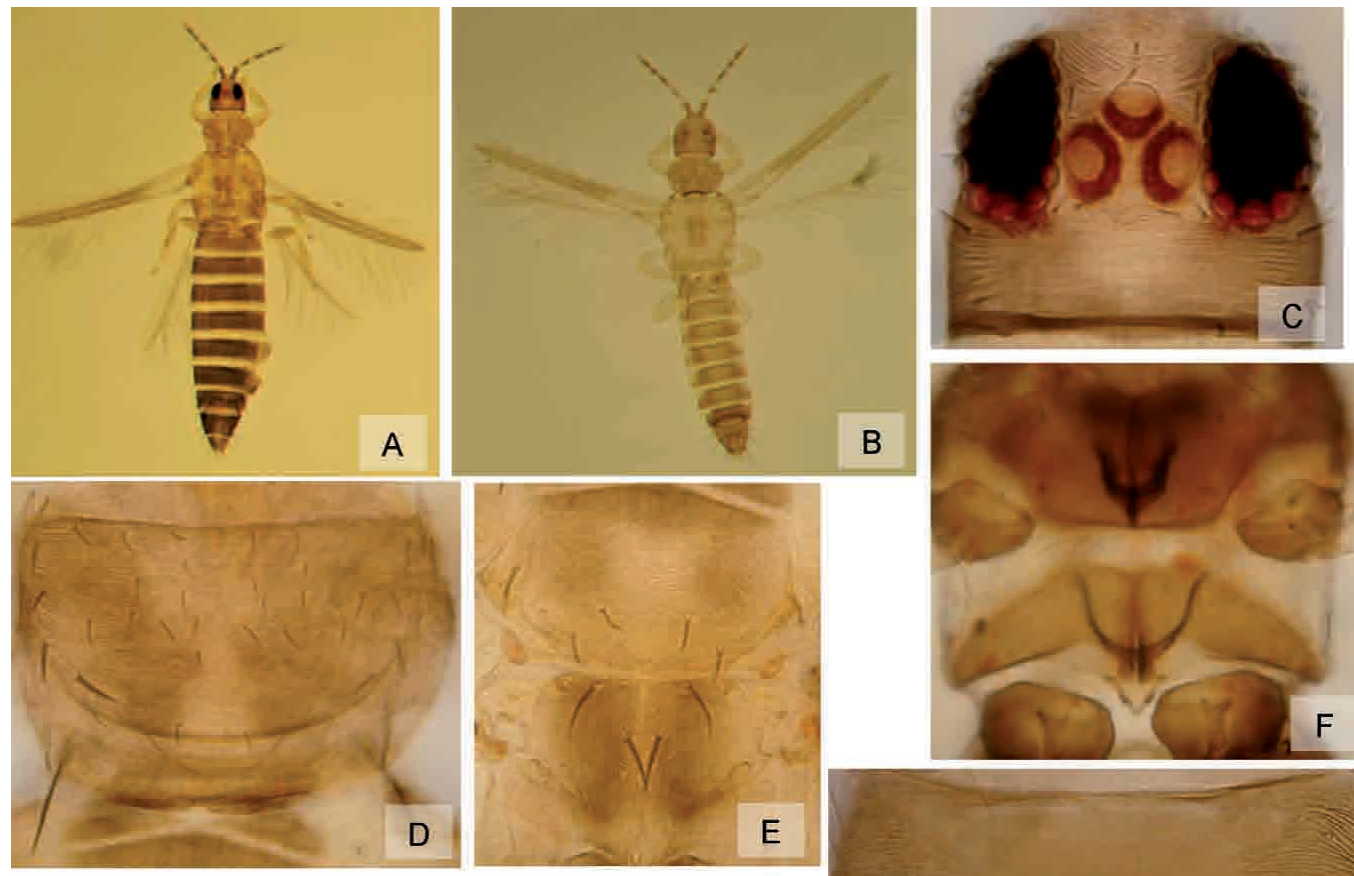

\section{B}
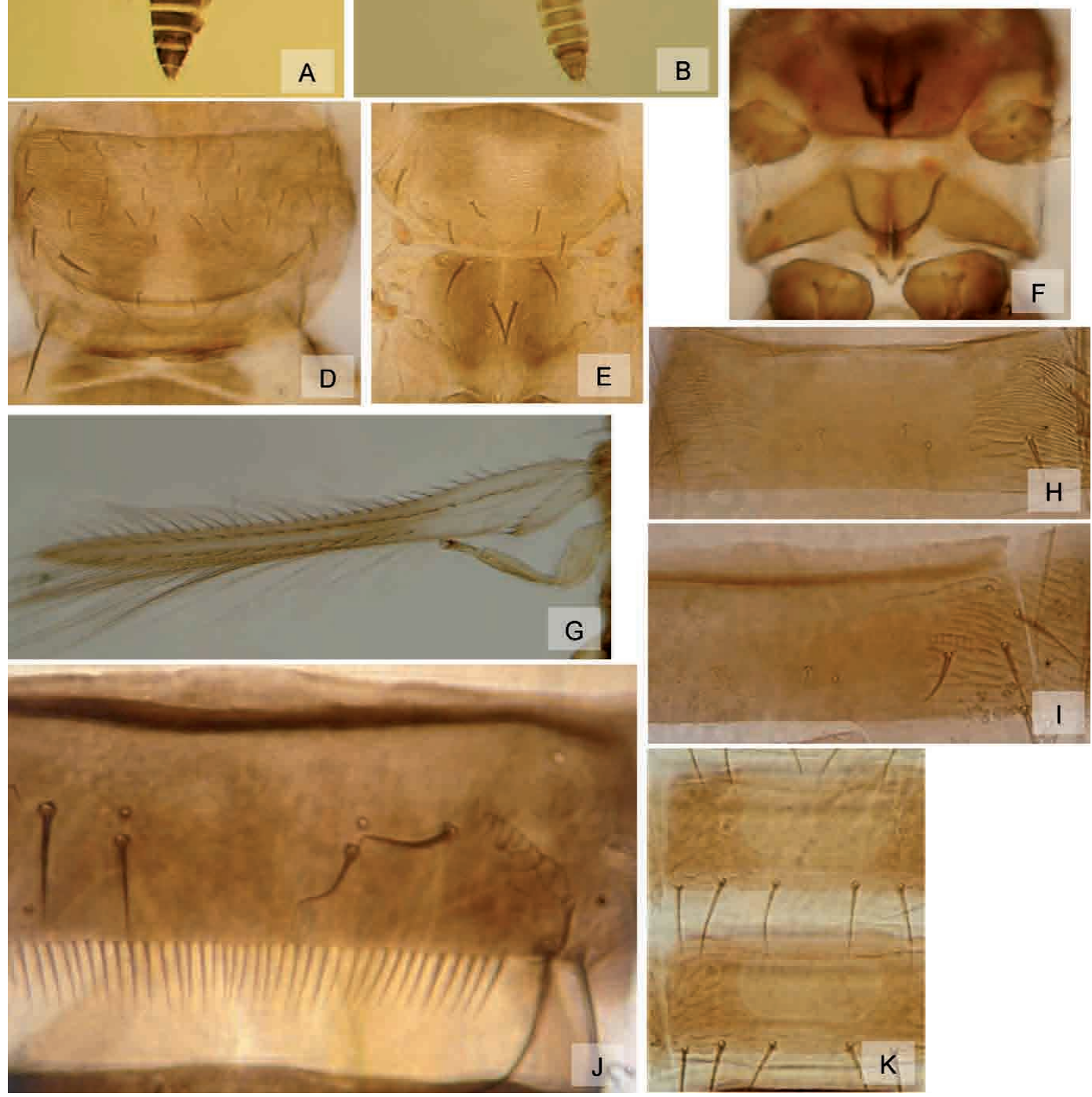

Figura 3. Chaetisothrips striatus. A: Hembra macróptera. B: Macho macróptero. C: Cabeza. D: Pronoto. E: Mesonoto y metanoto. F: Furcas mesoesternal y metaesternal. G: Ala anterior. H: Tergo abdominal III. I. Tergo abdominal IV. J: Peine del segmento VIII. K: Placas porosas de los esternos $\mathrm{V}$ y $\mathrm{VI}$ del macho. Las figuras 16 y 18-25, corresponden a la hembra. Autor: M. I. Zamar (octubre 2017). 
ticulados transversalmente, con tres pares de setas marginales posteriores, sin setas discales.

Macho macróptero: semejante a la hembra (Fig. 3B); con una placa porosa bien desarrollada en los esternos III a VII (Fig. 3K).

Plantas hospederas.- Al presente no existen referencias sobre la biología de esta especie. Fue obtenida a partir de especies de plantas de distintas familias como, $P$ rana paniculata Roxb. (Convolvulaceae) en Jamaica, Cassia moschata Kunth (Fabaceae) en Panamá y de Chioccoca alba (Rubiaceae) al sur de Brasil (Mound y Marullo, 1996; (Mound, Cavalleri, Lindner, Botton, de Souza Mendonça, 2018) En este estudio se cita por primera vez a Ch. striatus para Argentina, recolectada del envés de hojas de Cestrum parqui (Solanaceae).

Material examinado.- Argentina, Jujuy, Ledesma, Yuto (2338'33'S, 64²8'18'O; 354 m s.n.m.), 10 hembras, 00022 a 00031 INBIAL; 5 machos 00032 a 0036 INBIAL, 08/VIII/2016; hojas de Cestrum parqui. Col. Silvia Tapia.

\section{SCIRTIDOTHRIPS HOOD}

Este género contiene a Scirtidothrips torquatus Hood, única especie neotropical registrada en Brasil y Costa Rica (Mound et al., 2018). Semejante a Scirtothrips, del que se diferencia por carecer de hileras de microtriquias discales en los tergos y esternos abdominales.

\section{SCIRTIDOTHRIPS TORQUATUS HOOD}

Hembra macróptera (Fig. 4A). Cuerpo de color amarillento, ocelos de base anaranjada, segmento antenal I, amarillo claro, segmentos II-VIII castaños, suavemente más claros en la base; alas anteriores suavemente coloreadas de castaño. Cabeza estriada, excepto en el triángulo ocelar; tres pares de setas ocelares bien desarrolladas, el par III ubicado fuera en la línea externa del triángulo; un par de setas postoculares debajo de los ocelos posteriores y una seta debajo de los ojos (Fig. 4B). Antenas formadas por ocho segmentos, III y IV con conos sensoriales bifurcados (Fig. 4C). Pronoto rectangular, estriado, excepto en unas zonas circulares u oblongas laterales, cuatro o cinco pares de setas cortas anteromarginales, un par de setas posteroangulares largas, tres pares de setas posteromarginales y setas discales distribuidas en la superficie (Fig. 4D). Mesonoto estriado, con líneas anastomosadas (Fig. 4E). Metanoto estriado debajo del margen anterior y reticulado en el resto de la superficie; sin sensilias campaniformes; setas medias ubicadas debajo del margen anterior (Fig. 4E). Alas anteriores estrechas y agudas, primera vea con la serie de setas incompleta en la mitad distal, donde se encuentran dos setas; segunda vena completa (Fig. 4F). Tergos y esternos abdominales finamente reticulados transversalmente; algunas líneas tergales laterales llevan microtriquias; márgenes posteriores laterales de los tergos con microtriquias conspicuas (Fig. 4G); setas tergales medias largas, próximas entre sí (Fig. 4G); peine del tergo VIII completo (Fig. 4H). Esternos sin setas discales (Fig. 4I).

Macho: no observado.

Plantas hospederas. - Al presente no existen referencias sobre la biología de esta especie. La descripción original se realizó sobre la base de tres hembras recolectadas en Brasil, sin referencias de la planta hospedera. Mound y Marullo (1996) la citan sobre Calliandra portoricensis (Jacq.) Benth. (Fabaceae) en Costa Rica. En este estudio se registra por primera vez a $S$. torquatus en Argentina, en hojas de "frutilla" (Fragaria $x$ ananassa Duch. (Rosaseae)) y en flores de "pacará" (Enterolobium contortisiliquum (Vell.) Morong (Fabaceae)).

Material examinado.- Argentina, Jujuy, El Carmen (24²4'05,24”S, 6503'34,03” O; 847 m s.n.m.), 1 hembra, 00037 INBIAL, 07/VI/2016; ex/hoja de Fragaria $x$ ananasa (Weston) Duchesne. Col. María Inés. Zamar; San Pedro (24ำ' 42,32"S, 6453'55,19" O; 643 m s.n.m.), 1 hembra, 00038 INBIAL, 08/III/2017, ex/ flores de Enterolobium 

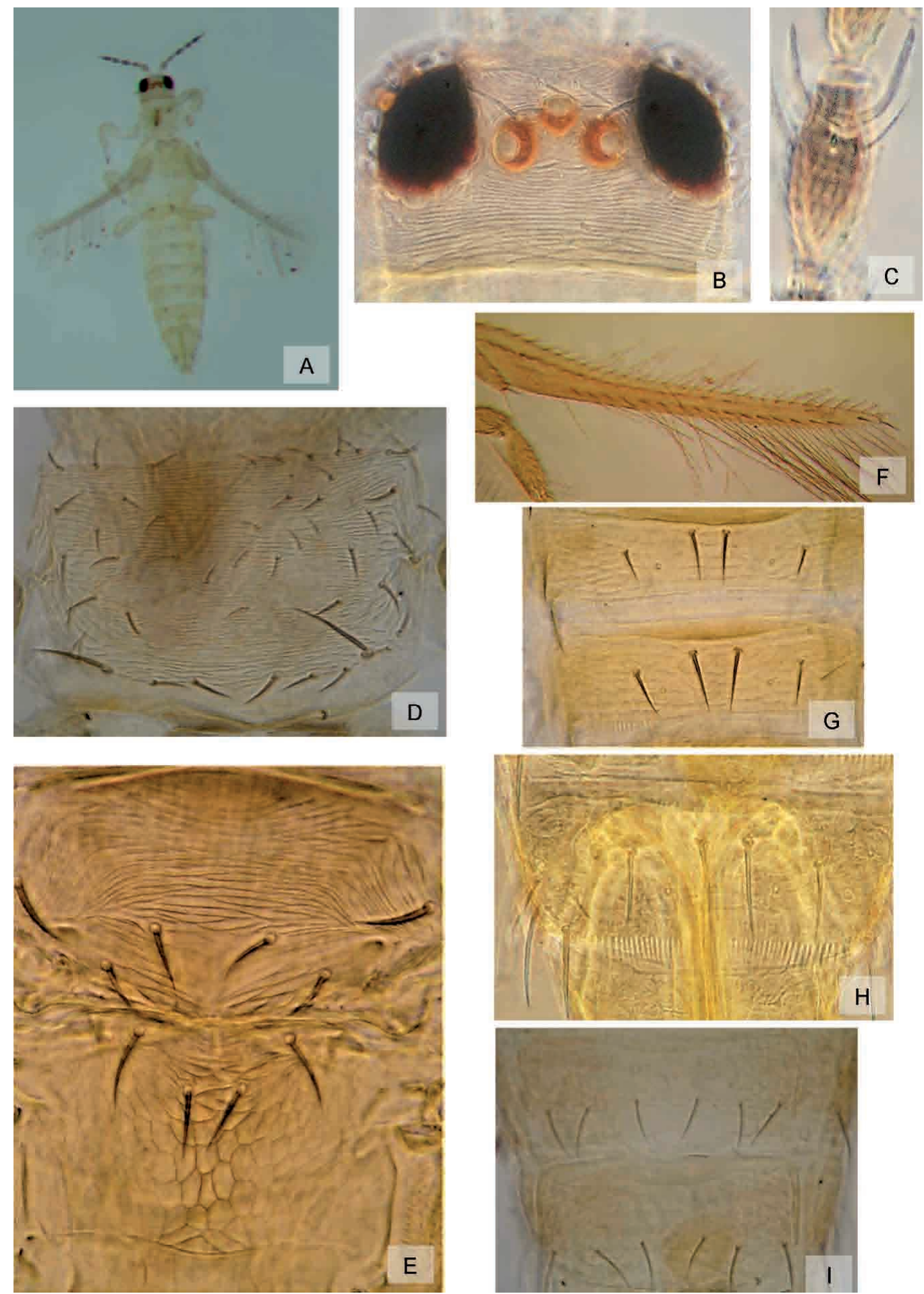

Figura 4. Scirtidothrips torquatus. A: Hembra macróptera. B: Cabeza. C: Cono sensorial del segmento antenal III. D: Pronoto. E: Mesonoto y metanoto. F: Ala anterior. G: Tergos abdominales IV y V. H: Peine del tergo abdominal VIII. I: Esternos abdominales VI y VII. Autor: M. I. Zamar loctubre 2017). 
contortisiliquum (Vell.) Morong. Col. María Inés Zamar.

\section{AGRADECIMIENTOS}

Al Dr. Élison Bezerra Lima (Universidade de São Paulo) y al Dr. Laurence Mound (CSIRO) por las orientaciones brindadas en la identificación de Ch. striatus y $S$. torquatus. A la Secretaría de Biodiversidad del Ministerio de Ambiente de la provincia de Jujuy, por el permiso de muestreo Res. $\mathrm{N}^{\circ} 171 / 2016$.

\section{FINANCIAMIENTO}

A la Secretaría de Ciencia y Técnica y Estudios Regionales de la Universidad Nacional de Jujuy por haber financiado parte de la investigaciones realizadas en este trabajo, a través del proyecto A- F0026.

\section{PARTICIPACIÓN}

La primera autora ha realizado las preparaciones microscópicas, identificaciones, obtenido las imágenes de las especies de trips, recolectado Scirtidothrips torquatus, ha redactado el manuscrito y realizado las correcciones sugeridas por los árbitros. La segunda autora y el tercer autor han participado mediante la recolección de trips de la provincia de Tucumán, la redacción del manuscrito y correcciones del texto final. Las dos últimas autoras han contribuido mediante la recolección de trips, redacción y corrección final del texto.

\section{CONFLICTOS DE INTERÉS}

En el presente trabajo no existen conflictos de interés entre los autores o con terceros.

\section{LITERATURA CITADA}

Buckman, R. S., Mound, L. A., Whiting M. F. (2013). Phylogeny of thrips (Insecta: Thysanoptera) based on five molecular loci. Systematic Entomology, 38, 123133.

Carrizo, B., Zamar, M. I. (2016). Tisanópteros (Insecta) presentes en flores de la vegetación espontánea frecuente en plantaciones de limón en Famaillá (Tucumán,
Argentina). Revista Agronómica del Noroeste Argentino, 36, 55-60.

Cavalleri, A., Piccoli Romanowski, H., Rodrigues Redaelli, L. (2006). Thrips species (Insecta, Thysanoptera) inhabiting plants of the Parque Estadual de Itapuã, Viamão, Rio Grande do Sul state, Brazil. Revista Brasileira de Zoologia, 23, 367-374.

Commonwealth Scientific and Industrial Research Organization. (2009). World Thysanoptera. http://anic.ento.csiro. au/thrips/identifying_thrips/Thripidae. $\mathrm{htm}$. Acceso [02/ 2018].

de Borbón, C. M. (2008). Desertahrips chuchiraga gen et sp. n. (Thysanoptera, Thripidae) from Argentina). Zootaxa, 1751, 25-34.

de Borbón, C. M. (2009). Tres nuevas citas de trips (Thysanoptera: Thripidae) para la Argentina y clave de los géneros de la familia Thripidae presentes en el país. Revista de la Facultad de Ciencias Agrarias UNCuyo, 41, 93-104.

de Borbón, C. M. [2013). Especies del género Frankliniella (Thysanoptera: Thripidae) registradas en la Argentina, una actualización. Revista de la Facultad de Ciencias Agrarias UNCuyo, 45, 259-284.

De Santis, L., De Sureda, A. E. G., Merlo, E. Z. (1980). Estudio sinóptico de los Tisanópteros Argentinos (Insecta). Obra del centenario de Museo de La Plata, 6, 91-166.

Goane, L., Pereyra. V., Salas, H. (2007). Presencia de Chaetanophothrips orchidii (Insecta: Thysanoptera: Thripidae) en fincas de limonero en Tucumán, Argentina. Revista Industrial y Agrícola de Tucumán, 84, 25-27.

Lima, B., Mound, L. A. (2016). Species-richness in Neotropical Sericothripinae (Thysanoptera: Thripidae). Zootaxa, 4162, $1-45$.

Mound, L. A., Marullo, R. (1996). The thrips of Central and South America: An introduction. Gainesville, Florida, USA: Memoirs on Entomology, International.

Mound, L. A., Cavalleri, A., Lindner, M. F., Botton, M., de Souza Mendonça, Jr., M. (2018). Os Tripes do Brasil. http:// www.thysanoptera.com.br/home. Acceso [12/2017].

Zamar, M. I. [2011]. La diversidad de thrips del Cono Sur. El caso de las zonas áridas en Jujuy, Argentina. Métodos en Ecología \& Sistemática, Costa Rica, 6, 71-88.

Zamar, M. I., Alejo, G., Gutiérrez, A. (2015). Dos citas nuevas de Thripidae (Thy- 
sanoptera) para la Argentina. En Actas IX Congreso Argentino de Entomología, Sección 2, 83. Misiones, Argentina.

Zamar, M. I., de Borbón, C. M., Aguirre, A.; Miño, V., Cáceres, S. (2014a]. Primer registro del daño de Leucothrips piercei (Morgan) [Thysanoptera: Thripidae) en cultivos de pimiento (Capsicum annuum L.) [Solanaceae) en la Argentina. Re- vista de la Facultad de Ciencias Agrarias Universidad Nacional de Cuyo, 46 , 213-219.

Zamar, M. I., Neder, L. E., Linares, M. A., Hamity, V. C., Contreras, E. F., Gómez G. (2014b). Tisanópteros (Insecta) asociados a plantas ornamentales de Jujuy (Argentina). Revista Agronómica del Noroeste Argentino, 34, 261-262. 